\title{
Severe aplastic anaemia in the Nordic countries: a population based study of incidence, presentation, course, and outcome
}

\author{
Niels Clausen, Anders Kreuger, Toivo Salmi, Ingebjørg Storm-Mathisen, \\ Gudmundur Johannesson
}

\begin{abstract}
Purpose-Incidence data for severe aplastic anaemia (SAA) in children are scanty and vary. Few population based studies have been reported. A retrospective and prospective study was conducted to determine the incidence and course of SAA.

Patients and methods-All children with a diagnosis of SAA in the Nordic countries from 1982 through 1993 were registered and have been followed up since 1987.

Results-A total of 101 children were diagnosed with SAA. The mean annual child population was 4.31 million. A constant incidence of $1.95 / \mathrm{million}$ children/year was found: 2.4 for boys and 1.5 for girls. A non-significant increase of cases occurred from November to March. Possible aetiological agents were noted in $29 \%$. The actuarial survival was $79 \%$ after one year and $68 \%$ after five years without significant difference between boys and girls.

Conclusion-The incidence of SAA in the Nordic countries remains stable with a preponderance among boys. SAA has still a high initial mortality and a risk of late deaths.

(Arch Dis Child 1996; 74: 319-322)
\end{abstract}

Keywords: aplastic anaemia, epidemiology, survival.

Children with acquired severe aplastic anaemia (SAA) as defined by the International Aplastic Anemia Study Group ${ }^{1}$ have a grave prognosis. The distinction between this disease complex and constitutional aplasias or neoplasias may be impossible at presentation. A period of observation may be needed before the diagnosis can be established. ${ }^{2}$ True incidence figures are dependent on reliable registrations. Furthermore, the index population must be steady and constantly surveyed by the health care system. The five Nordic countries, Denmark, Finland, Iceland, Norway and Sweden, have a stable population served by a uniform health system, which makes them suited for studies of incidence, exposures to different agents, and course of diseases.

The aim of the study was to determine the incidence and course of SAA and to investigate the frequency of different aetiological agents.

Patients and methods

All children under the age of 15 years, who were diagnosed with hypoplastic cytopenia from 1 January 1982 to 31 December 1993 in the five Nordic countries were registered and a follow up was done in April 1994.

The registration started in 1987, and was thus retrospective during the first five years and prospective since then. In the Nordic countries, care for severe aplasia is provided only through hospitals and not in private practice. All departments of paediatrics and departments of internal medicine admitting children were included in the investigation. They had a set of uniform investigations and report forms. A panel of haematologists was established for consultation in difficult cases. A yearly inquiry of diagnosis and follow up data has been performed since 1987. All cases were ascertained through hospital records and explicitly investigated for possible exposure to infectious and chemical agents, including drugs.

Patients with SAA were identified when at least two of three peripheral blood criteria were fulfilled: granulocyte count of less than $0.5 \times 10^{9} / 1$, platelet count less than $20 \times 10^{9} / 1$, and reticulocyte fraction less than $1 \%$ corrected for packed cell volume (PCV) (that is, $\times$ (actual PCV/normal PCV for age)) with the bone marrow having less than $25 \%$ normal cellularity or a marrow of less than $50 \%$ cellularity with less than $30 \%$ haematopoietic cells. Children with fewer than $0.2 \times 10^{9} / 1$ granulocytes had very severe aplastic anaemia (VSAA). When progression to a haematological malignancy occurred within three months the child was considered to have had preleukaemia initially and was not included in the study.

The mean annual population of boys and girls under 15 years of age was obtained from the Central Statistical Bureaus in each of the Nordic countries.

\section{STATISTICAL ANALYSIS}

Survival curves were calculated by the method of Kaplan and Meier ${ }^{3}$ with and without stratification for the following variables as reported at the time of diagnosis: age, sex, and treatment given. Data were also analysed by log rank test.

\section{Results}

A total of 101 children, 64 boys and 37 girls, under 15 years of age (range $1-14.8$ years, median 8.7 years) were diagnosed with SAA in the Nordic countries in the years 1982-93. 
Table 1 Number of cases of $S A A$, population under 15 years, and incidence of $S A A$ in the Nordic countries in 1982-93

\begin{tabular}{lrrrrrr}
\hline & Denmark & Finland & Iceland & Norway & Sweden & $\begin{array}{l}\text { Nordic } \\
\text { countries }\end{array}$ \\
\hline SAA & & & & & & \\
Boys & 10 & 16 & 3 & 9 & 26 & 64 \\
Girls & 6 & 12 & 0 & 9 & 10 & 37 \\
$\quad \begin{array}{l}\text { Total } \\
\text { Population }\end{array}$ & 16 & 28 & 3 & 18 & 36 & 101 \\
Boys & 469645 & 489691 & 32525 & 422969 & 790279 & 2205109 \\
Girls & 448900 & 468148 & 31024 & 402719 & 751368 & 2102159 \\
$\quad \begin{array}{l}\text { Total } \\
\text { Incidence }\end{array}$ & 918545 & 957839 & 63549 & 825688 & 1541647 & 4307268 \\
Boys & 1.8 & 2.7 & 7.7 & 1.7 & 2.7 & 2.42 \\
Girls & 1.1 & 2.1 & 3.9 & 1.9 & 1.1 & 1.47 \\
Total & 1.5 & 2.4 & 3.9 & 1.8 & 2.0 & 1.95 \\
\hline
\end{tabular}

The mean annual population under 15 years was 4.31 million. The overall incidence of SAA among Nordic children was $1 \cdot 95 /$ million/year. Figures for boys and girls in each country are given in table 1 .

When the incidence in the first half period, which comprised 50 cases (1.96/million/year), was compared with that of the last period with 51 cases ( $1 \cdot 99 / \mathrm{million} / \mathrm{year})$, no significant difference was found.

The most common symptom leading to the first contact with a doctor was haemorrhagic diathesis ( 49 children). It was mild in 50 children, prominent in 35, and seven had visceral bleeding at presentation. Other symptoms leading to the first consultation were fatigue in 34 children and infection in 18.

The characteristic child presenting with SAA was a child of normal growth, without malformations, previous severe disease, or hereditary diseases in first degree relatives. Three fourths of the group had heights within $1 \mathrm{SD}$ for age. In 86 children there were no diseases reported in first degree relatives. Three children had been adopted, and were thus without any known family history. In 12 children with a positive family history there were two siblings with SAA, one child with a sibling with immune thrombocytopenia, and two children with parents with haematological malignancies. Three had first degree relatives with allergies. Three parents had systemic lupus erythematosus, ulcerative colitis, and diabetes mellitus, respectively. One child's mother and her family had cholinesterase deficiency. This survey indicated an over-representation of cases of bone marrow hypoplasia and malignancy in first degree relatives.

Six of the 101 children had congenital defects. Five were cardiovascular, that is, transposition of the great arteries, persistent ductus arteriosus, ventricular septum defect, and lastly a combined ventricular septum defect and pulmonary stenosis. One child had skeletal defects. No other organ defects were noted in the patients.

The majority of the children had previously been healthy. In five children allergy was noted, one child had migraine, one petit mal, and one had seizures. Three children had previously had severe infections such as intracranial abscess, recurrent otitis, and pyelonephritis. Other reported conditions were Down's syndrome, circumscript scleroderma, hip dysplasia, diabetes mellitus, and submersion. There was no reported relation between the disease or its treatment and subsequent development of SAA in any of the cases.

Signs and symptoms had been present for less than a month in $67 \%$ and the diagnostic criteria were fulfilled at first presentation in $75 \%$. A gradual evolvement of cytopenia occurred in the remaining 27 children always including thrombocytopenia from the beginning. Twelve patients had additional erythropenia, and nine also had neutropenia. The longest period of evolvement from cytopenia to overt SAA was 60 months in one child, while the rest had a period from 0.5 to 16 months, median 2 months.

VSAA, that is $<0 \cdot 2 \times 10^{9} / 1$ neutrophils, was diagnosed in 53 children, and 87 children fulfilled the neutrophil criterion $<0.5 \times 10^{9} / 1$. Two had normal neutrophil counts. Only four children did not fulfil the thrombocyte criterion. Their platelet count was $21,25,29$, and $52 \times 10^{9} / 1$, respectively. Reticulocytes were below $1 \%$ in all but 10 children. In 74 children, all three peripheral blood count criteria were fulfilled.

During the winter from November to March more cases were diagnosed than in the summer season as illustrated in fig 1 . The median diagnostic delay from the first symptom or sign to the diagnosis was only one month, ranging from 0 to 68 months. This suggests, that most cases of SAA develop in the winter.

A possible cause of the SAA was reported to be chemicals in 24 children and infections in 11 as described in table 2 .

Three children died before start of treatment, and three were observed without treatment awaiting a bone marrow transplantation. No common treatment protocol was followed, but a bone marrow transplantation was performed when a compatible donor was available.

Treatment data are given in table 3.

A total of 30 children have died. Median survival time was 8 months (range 12 days to 89 months) after the diagnosis. The cause of death was sepsis in 17 including five proved cases of fungal sepsis, complications after bone marrow transplantation in five, intracranial bleeding in five, and a profuse bleeding because of disseminated intravascular coagulation in three.

The actuarial overall survival of the children after one year was $79 \%$ (95\% confidence interval (CI) 70 to 86 ), and after five years it

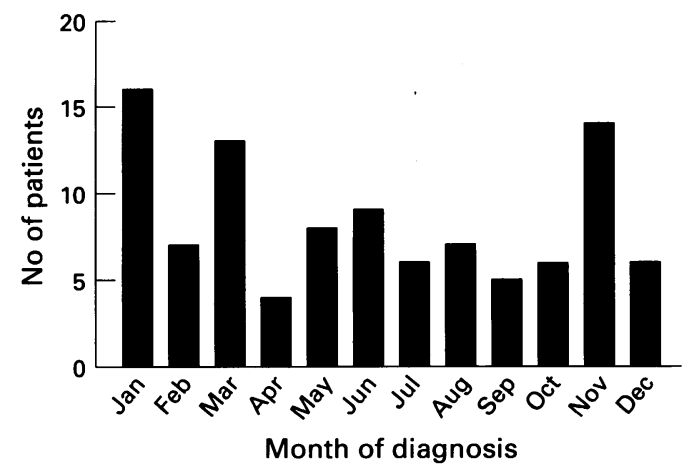

Figure 1 Number of cases diagnosed each month of the year. 
Table 2 Exposure to chemical and infectious agents in 31 children; four had double exposure, which is indicated by superscript 1-4 (number of patients in parentheses)

Drugs

Penicillin, erythromycin (3), chloramphenicol, trimethoprim-sulphamethoxazole (2), nitrofurantoin, viprynium embonate, ethosuximide, clonazepam/phenobarbitone, imipramine ${ }^{1}$, ammonium chloride, measles, mumps, rubella vaccine ${ }^{2}$

- Organic diluents

Xylene ${ }^{3}$, turpentine ${ }^{4}$, alkyd paint ${ }^{1}$, herbicides

- Glue $^{34}$

- Cadmium

- Hepatitis

Hepatitis A and B antibody negative (6), hepatitis A antibody positive (2), hepatitis, antibody reaction unknown ${ }^{2}$ (1)

- Mononucleosis

Epstein-Barr virus IgM titre raised

- Other viral infections

Parvovirus IgM titre raised at diagnosis was $68 \%$ (95\% CI 58 to 77$)$. Survival curves for boys and girls are given in fig 2 . There was no difference between the survival in children with a known aetiology to the SAA, and the idiopathic cases $(\log$ rank $p=0 \cdot 81$ ). The crude survival rate of children treated with bone marrow transplantation was $83 \%$, compared with $65 \%$ of the non-transplanted (log rank $\mathrm{p}=0.07$ ).

As of 1 April 1994 the 71 surviving children showed a median survival time of 70 months. A total of 52 were in complete remission and had finished treatment, 25 after allogeneic bone marrow transplantation. The median time to complete recovery of the platelet count was 266 days for girls and 1003 days for boys under 10 years, while over the age of 10 years the time was 137 days and 425 days, respectively. Thus, slow recovery was related to young age and male gender.

Three children had relapse of SAA after complete remission had been obtained for four, four, and eight months, respectively. Complete remission had not been obtained in 19 children after a median survival of 72 months after the diagnosis.

Three children had sequelae (two impaired renal function due to cyclosporin, one chronic graft versus host reaction). One child got myelodysplasia with excess of blasts after 47 months.

\section{Discussion}

An annual incidence of SAA of $1.95 /$ million in children was found in the Nordic countries. The figure was steady during the 10 year period. The variation between the figures from the different countries was not significant. Previous studies of SAA in children from single Nordic countries showed incidences in Denmark of 2.2 in $1967-82$, which is within

Table 3 Treatment data indicating drugs, doses, and number of patients responding/total number treated; some patients received more than one treatment schedule

Allogeneic bone marrow transplantation

$25 / 30$

Methyltestosterone 3-5 mg/kg/day orally and glucocorticosteroids

Highdose immunoglobulin

Glucocorticosteroids and antithymocyte globulin $20 \mathrm{mg} / \mathrm{kg} /$ day intravenously, ${ }^{4}$ median 8 days

Cyclosporin $9 \mathrm{mg} / \mathrm{kg} /$ day orally, ${ }^{5}$ median 90 days Antithymocyte globulin and cyclosporin

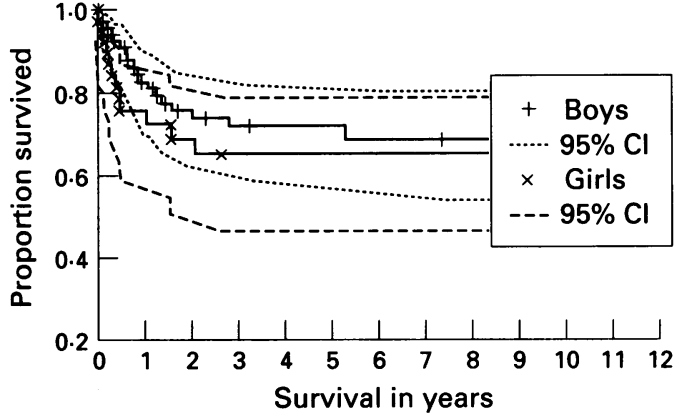

Figure 2 Kaplan-Meier survival estimates in 64 boys and 37 girls.

the range of the present results. In a Swedish region the annual incidence of both SAA and less severe aplastic anaemia in children was 4-5/million children in $1964-8 .^{6}$

In a study from 1984-7 in 83 university affiliated medical centres in France, serving mainly metropolitan populations, the incidence of SAA in children was 1.28 for boys and 0.88 for girls giving a figure of 1.0 for all. ${ }^{7}$ A regional study in 1985 in the UK found $1.3 /$ million males and 0.7 for females under 20 years of age. ${ }^{8}$ In metropolitan Baltimore, 15 children aged 0-19 years with moderate aplastic anaemia and SAA were identified from the years 1970 through 1978 . This gave an average annual incidence of $4 \cdot 6 /$ million boys and $1.7 /$ million girls. The cases were retrieved through hospital charts and death certificates. It is notable, however, that $34 \cdot 7 \%$ had only a death certificate and no hospital record. ${ }^{9}$ A study in Bangkok found $2 \cdot 4$ cases of moderate aplastic anaemia and SAA per million children under 15 years with 3.8 males and 0.8 females. It was not stated how many cases had SAA. ${ }^{10}$

Even when identical diagnostic criteria for SAA are employed, there are variations in incidence figures from different countries. Low figures may clearly be due to incomplete retrieval of cases. In the present study the surveillance method warrants supposedly complete reporting, as the socialised medical care in the Nordic countries ensures referral to a hospital centre of all cases of aplastic anaemia and reporting of the diagnosis to the central medical authorities. Verification of the diagnosis was done by the study centre based on data from the hospital records. An important check was provided through the long term follow up which ruled out cases of paroxysmal nocturnal haemoglobinuria and myelodysplasias.

Few prospectively designed studies have evaluated the incidence of SAA. Even prospective studies have not been truly population based. In a French prospective study the number of participating centres increased during the study period. ${ }^{7}$ A prospective Thai study comprised only cases dealt with by acute care institutions with at least 100 beds. ${ }^{10}$ In the present multinational, complete patient cohort no difference in incidence was found between the groups analysed retrospectively or prospectively.

The slight non-significant seasonal variation in incidence with higher figures in November 
to March than from July to October substantiates previous findings in Denmark. ${ }^{11}$ The same trend was found in France, ${ }^{7}$ whereas in the UK the numbers were higher from May to October. ${ }^{8}$ A seasonal variation could be due to epidemics of infections or to exposure to certain chemicals as drugs, pesticides, and herbicides. The registration of the date of diagnosis rather than the time of the first symptom may blur a marked seasonality in exposure since the speed with which the disease evolves may vary. However, most cases $(67 \%)$ had a sudden onset and less than one month from the first sign or symptom to the diagnosis.

Other epidemiological studies on SAA have not focused on the premorbid status of the patients. We found that the children were essentially normal concerning growth, previous diseases and disposition to allergies, whereas dispositions to blood diseases and haematological malignancies were over-represented. Cartwright et al found a cluster of five cases who all were atopic. ${ }^{8} \mathrm{We}$ found only five children with allergy out of 101 , which is within normal limits. Congenital heart disease was significantly over-represented in this study with five cases in 101 of the SAA patients. Normally, the prevalence of congenital heart disease is less than $1 \%$. There is no evidence to support the idea that non-constitutional cases of SAA develop in susceptible children due to allergy or congenital abnormalities, although the disease may be caused by a perturbed immune function. The perturbation may be caused by external factors, and it may persist even when normal peripheral blood counts have been reestablished by immunosuppressive treatment. ${ }^{12}$

Initially the platelet count will nearly always be affected. This occurs in cases with both sudden and gradual onset. ${ }^{13}$ The second most often affected cell line is the erythrocyte line. Normal reticulocyte counts were seen in only $1 \%$, whereas the neutrophil count was normal in $2 \%$.

The platelet count normalised slower than that of neutrophils and erythrocytes in both boys and girls in the present study. A Swiss study found that a group of six girls under 10 years of age had a significantly slower platelet recovery. ${ }^{14}$ The comparable group in the present study comprised 17 girls with a median recovery time, which was no longer than that of the boys. Both boys and girls aged less than 10 years had a longer recovery time than older children.

A wide variety of agents has been suspected of causing SAA. This study found a probable aetiological agent in the history of 31 children $(31 \%)$. Other cohort studies have indicated aetiological agents in $50 \%{ }^{11}$ The incidence of drugs as a single cause of SAA has been reported to be as high as $50 \%, 15$ and drug induced aplasias in the elderly account for a substantial number. ${ }^{16}$

Girls tend to have a higher mortality than boys during the first year, but due to late deaths among boys the survival curves converge later $(\log$ rank $p=0.43)$. This is in agreement with the finding of a slow recovery of neutrophil counts during the first year in girls compared with all other patients. ${ }^{14}$

The actuarial survival from SAA of $79 \%$ after one year and $68 \%$ after five years compares favourably to that found in other population based studies. The French study of both moderate and severe cases showed a fatality rate after one year of $34 \% .{ }^{7}$ Thus, SAA is still a disease with a high initial mortality and a risk of late relapse and death.

We thank Professor M Seip for keen participation in data collection in Norway.

1 Camitta BM, Thomas ED, Nathan DG, et al. A prospective study of androgens and bone marrow transplantation for treatment of severe aplastic anemia. Blood 1979; 53: 504-14

2 Matloub YH, Brunning RD, Arthur DC, Ramsay NK Severe aplastic anemia preceding acute lymphoblastic leukemia. Cancer 1993; 71: 264-8.

3 Kaplan EL, Meier P. Non-parametric estimation for incomplete observations. Fournal of the American Statistical Association 1958; 53: 457-60.

4 Bayever E, Champlin R, Ho W, et al. Comparison between bone marrow transplantation and antithymocyte globulin in treatment of young patients with severe aplastic in treatment of young patients

5 Seip M, Vidnes J. Cyclosporin A in a case of refractory severe aplastic anemia. Scandinavian fournal of Hematology 1985; 34: 228-30.

6 Böttiger LE, Westerholm B. Aplastic anaemia. I. Incidence and aetiology. Acta Paediatr Scand 1972; 192: 315-8.

7 Mary JY, Baumelou E, Guiguet M. Epidemiology of aplastic anemia in France: a prospective multicentric study. Blood 1990; 75: 1646-53.

8 Cartwright RA, McKinney PA, Williams L, et al. Aplastic anaemia incidence in parts of the United Kingdom in 1985. Leuk Res 1988; 12: 459-63.

9 Szklo M, Sensenbrenner LL, Markowitz JA, Weida S. Warm S, Linet MS. Incidence of aplastic anemia in metropolitan Baltimore: a population-based study. Blood ropolitan Baltim

10 Issaragrisil S, Sriratanasatavorn C, Piankijagum A, et al. Incidence of aplastic anemia in Bangkok. Blood 1991; 77: 2166-8.

11 Clausen N. A population study of severe aplastic anemia in children. Incidence, aetiology and course. Acta Paediatr Scand 1986; 75: 58-63.

12 Camitta BM, Doney K. Immunosuppressive therapy for aplastic anemia: indications, agents, mechanisms and results. Am f Pediatr Hematol Oncol 1990; 12: 411-24.

13 Khatib Z, Wilimas J, Wang W. Outcome of moderate aplastic anemia in children. Am $\mathcal{F}$ Pediatr Hematol Onco 1994; 16: 80-5.

14 Nissen C, Gratwohl A, Tichelli A, et al. Gender and response to antilymphocyte globulin (ALG) for severe aplastic anaemia. Br f Haematol 1993; 83: 319-25.

15 Malkin D, Koren G, Saunders EF. Drug-induced aplastic anemia: pathogenesis and clinical aspects. Am $\mathcal{f}$ Pediat Hematol Oncol 1990; 12: 402-10.

16 Baumelou E, Guiguet M, Mary JY. Epidemiology of aplastic anemia in France: a case-control study. I. Medical history and medication use. The French Cooperative Group for Epidemiological Study of Aplastic Anemia. Blood 1993; 81: 1471-8. 